\title{
A STUDY OF THE LOCALIZATION OF FLARES IN SELECTED ACTIVE REGIONS
}

\author{
M. J. Martres, R. Michard, I. Soru-Iscovici, T. Tsap \\ (Observatoire de Paris-Meudon, France and Crimean Astrophysical Observatory \\ Nauchny, Crimea, U.S.S.R.)
}

\begin{abstract}
A BSTRACT
From the material gathered during the 'Cooperative Study of Solar Active Regions', we studied the flare locations in AR magnetic structure, and flare relations to changes in the magnetic fields and spot configurations. Besides a confirmation of previous results, we find that flares are often associated with two features of the spot configuration evolving in opposite senses, one growing, the other declining.
\end{abstract}

Previous work has shown that flares tend to occur at privileged locations in the magnetic configuration of solar AR. Severny $(1958,1963)$ pointed out that most flares start near the line of separation of polarities of the longitudinal field, specially if the horizontal 'gradient' of $H_{\|}$is strong. It was soon recognized that this also means a strong transversal field (Michard et al., 1961). Later on Severny emphasized the significance of points of 'bifurcation' of lines of force as a seat of flares (Severny, 1964). Finally Martres et al. (1966) pointed out that in case a flare is made up of two (or more) bright features these seem to appear at places of opposite polarities.

As flares show preferred locations in space, they also show preferred locations in time, being obviously associated with AR which are changing rapidly from the point of view of spot area (Giovanelli, 1939), spot configuration (Gopasyuk et al., 1963) and therefore also magnetic structure. It seems that the pre-flare magnetohydrodynamic configuration in the chromosphere and corona is not an unstable one, but has to be destroyed by a finite change in the photospheric field: in other words, it is a metastable configuration.

Accordingly the 'location' of flares should be studied simultaneously from both an historical and topographical point of view; a rather difficult task, which has not been attempted so far.

Some of the AR selected for the CSSAR project had a fair production of flares. Thanks to the generous cooperation of the participating observatories, it was possible to collect in Meudon:

(1) a continuous sunspot history with about one picture per hour,

(2) a nearly continuous $\mathrm{H} \alpha$ cinematographic record for days of significant flare activity, 
(3) fairly complete series of calcium spectroheliograms,

(4) a number of detailed magnetic maps of AR from K.A.O. and Meudon, which, although significantly larger than hitherto achieved, proved still insufficient in many cases.

The study of this material from the point of view of flare location in space and time was started in Meudon rather recently, and only preliminary results can be given here. Various approaches to the problem were tried in succession.

\section{1st approach:}

This consisted in comparing the $\mathrm{H} \alpha$ pictures of flares with the magnetic maps obtained on the same day (and as close as possible in time), in order to find the location of the starting-points of flares. In this comparison, a necessary step is to locate correctly sunspots on both the magnetic and the $\mathrm{H} \alpha$ pictures: errors of 5 to $10^{\prime \prime}$ are not unusual, specially when the time interval of both observations exceeds 2 hours.

Such comparisons were performed for 36 flares of regions CSSAR 4, 8, 9 and 10, and a statistic was made of the distances to the inversion lines of $H_{\|}$of 154 bright points associated with the flares: in $40 \%$ of the cases these points are practically on the inversion line, while in $67 \%$ their distance is $\leqslant 10^{\prime \prime}$. We found two exceptional cases, two flares which contained only a single bright point far away from any inversion line. However, the magnetic map had been obtained 6 to 7 hours before the flares: the comparison with the magnetic data of the following day showed that a new inversion line had developed at some time between the two observations, possibly before the flares themselves.

The tendencies for flares to occur at places of strong horizontal gradient of $H_{\|}$ was confirmed. Also it was confirmed that if more than one bright point occurs (31 cases out of 36) they are distributed on both sides of the inversion line: exceptions to this rule were few and occurred when the time interval between the magnetic observation and the flare was large.

Examples of flare locations are given in Figure 1.

\section{2nd approach:}

An attempt was made to trace quantitative changes of the fields and to relate these to flare occurrence. For this purpose, the integrals $F_{\mathrm{N}}$ and $F_{\mathrm{S}}$ were calculated.

We have

$$
F_{\mathrm{N}}=\int_{A} H_{\mathrm{mN}} \mathrm{d} S
$$

where $H_{\mathrm{mN}}$ is the measured longitudinal field of $\mathrm{N}$ polarity, $S$ is the element of area of the solar photosphere, and $A$ is the explored part of the Sun. Further we calculated the indices

$$
\Sigma=\left|F_{\mathrm{N}}\right|+\left|F_{\mathrm{S}}\right| \quad \text { and } \quad k=\frac{\left|F_{\mathrm{N}}\right|-\left|F_{\mathrm{S}}\right|}{\Sigma} .
$$




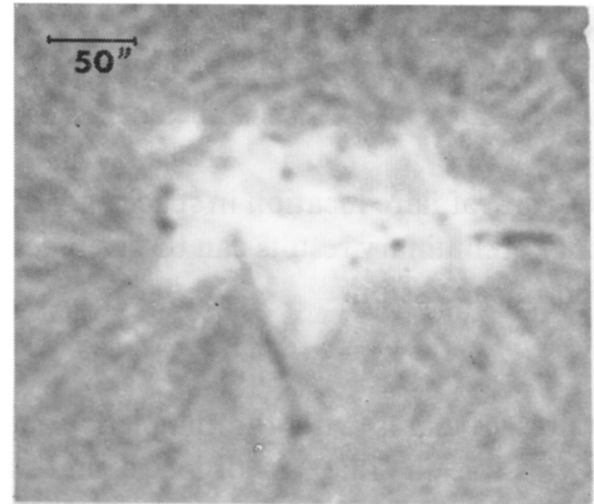

$\mathbf{a}_{1}$

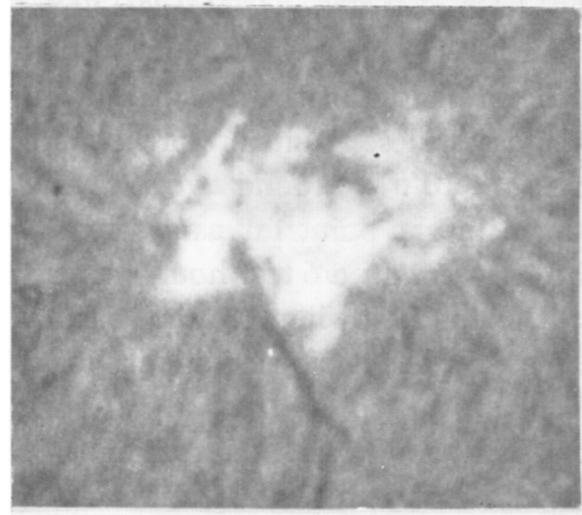

$\mathbf{b}_{1}$

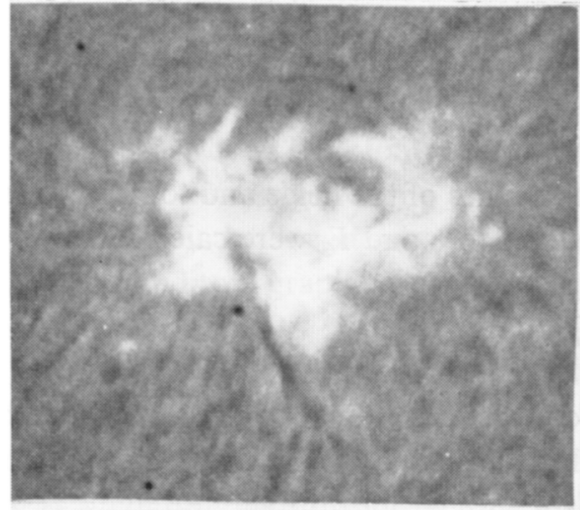

$c_{1}$

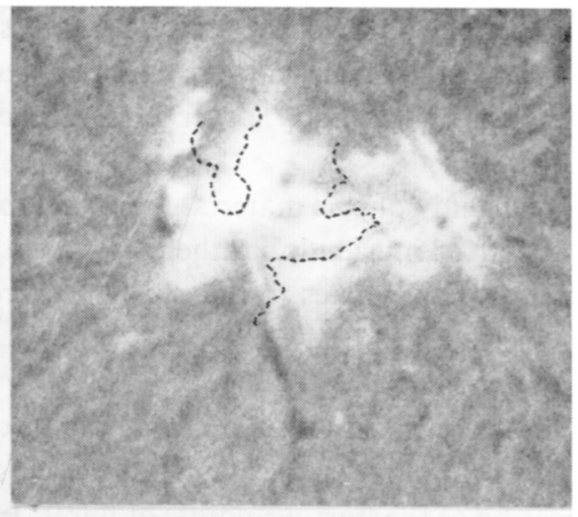

$\mathbf{a}_{2}$

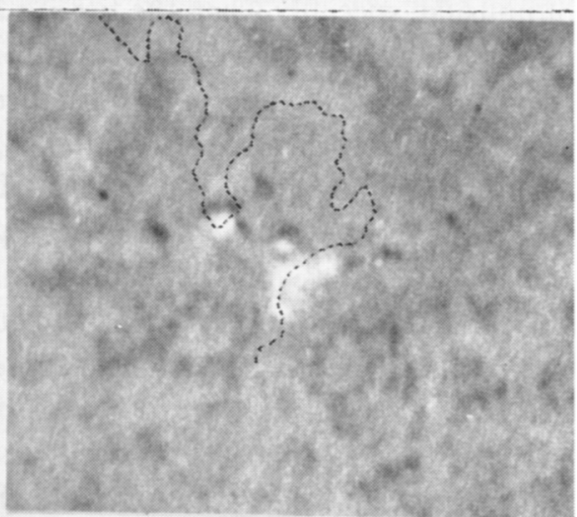

$\mathbf{b}_{2}$

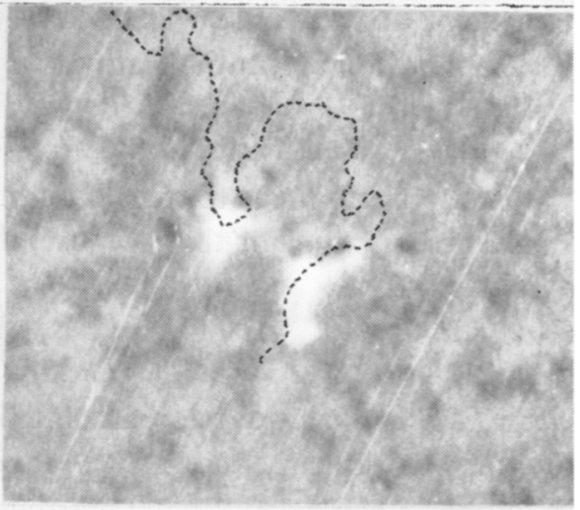

$\mathbf{c}_{2}$ 
In cases where the AR is at the centre of the disk, where the measured field equals the longitudinal field and where $A$ is sufficiently large, we could more or less identify $F_{\mathrm{N}}-F_{\mathrm{S}}$ with the flux of $\mathbf{H}$ through a closed surface and expect it to be zero. These three conditions are never fulfilled: the calculated quantities $\Sigma$ and $k$ are nothing more than possibly useful indices of total field strength and distribution.

Figure 2 gives the variation of $\Sigma$ and $k$ for the region CSSAR 9 between September 28 and October 5, 1965. Homogeneous magnetic data from K.A.O. was available and could be supplemented by Meudon data using an empirical relation between $H_{\mathrm{m}}$ (K.A.O.) and $H_{\mathrm{m}}$ (Meudon). It seems that flare occurrence was more frequent when the index $\Sigma$ was large and the index of 'unbalance' $k$ rapidly changing (see other comments in figure caption).

On the other hand the same indices where evaluated for these cases where two magnetic maps were available, one before, the other after a flare (interval 2-5 hours). Six such cases were found in the CSSAR material and two more were added from publications by Severny (1960) and Gopasyuk et al. (1963).

In this material there were indications that $\Sigma$ decreased after flares: 6 cases out of 8 , and that $k$ changed, indifferently decreasing ( 3 cases) or increasing ( 5 cases).

The significance of these results will have to be tested by the study of more and better observations, and by objective evaluations of errors in the determination of the used indices. We believe that in about half the 8 cases under study, the changes of $\Sigma$ and $k$ following a flare did not exceed possible errors in the evaluation of these parameters, so that we cannot conclude.

\section{3rd approach:}

In this case it was tried to relate flare occurrence to the geometry and evolution of sunspot groups. With the CSSAR material it is possible to follow sunspot evolution in a fairly continuous way. Sunspots represent the distribution of peaks of the total field strength (not the longitudinal component). Their magnetic polarities are often known from patrol visual observations, even when no detailed field maps are available. Therefore good sunspots observations are a very useful substitute to the present incomplete magnetic observations.

The study of an AR like CSSAR 7 showed that new sunspots are continuously born

FIG. 1. Example of location of flares relative to the inversion line of longitudinal field: region CSSAR 9, October 2, 1965. In each case a pre-flare Ha picture (1) and a picture during the flare (2) are given. Case (a): Magnetic map from K.A.O. obtained 0735-1000 UT. Ho images from Culgoora at 0350 and 0413. Case (b): Magnetic map from K.A.O. obtained 1230-1410 UT. Ha images from Sacramento Peak at 1338 and 1414 (off-band picture). Case (c): Same magnetic data as in (b). Ha images from Sac. Peak at 1555 and 1620 (off-band picture). - Note that the fit of flare knots and filaments to the inversion line is the best when the time interval between magnetic observations and fare is the shortest. 


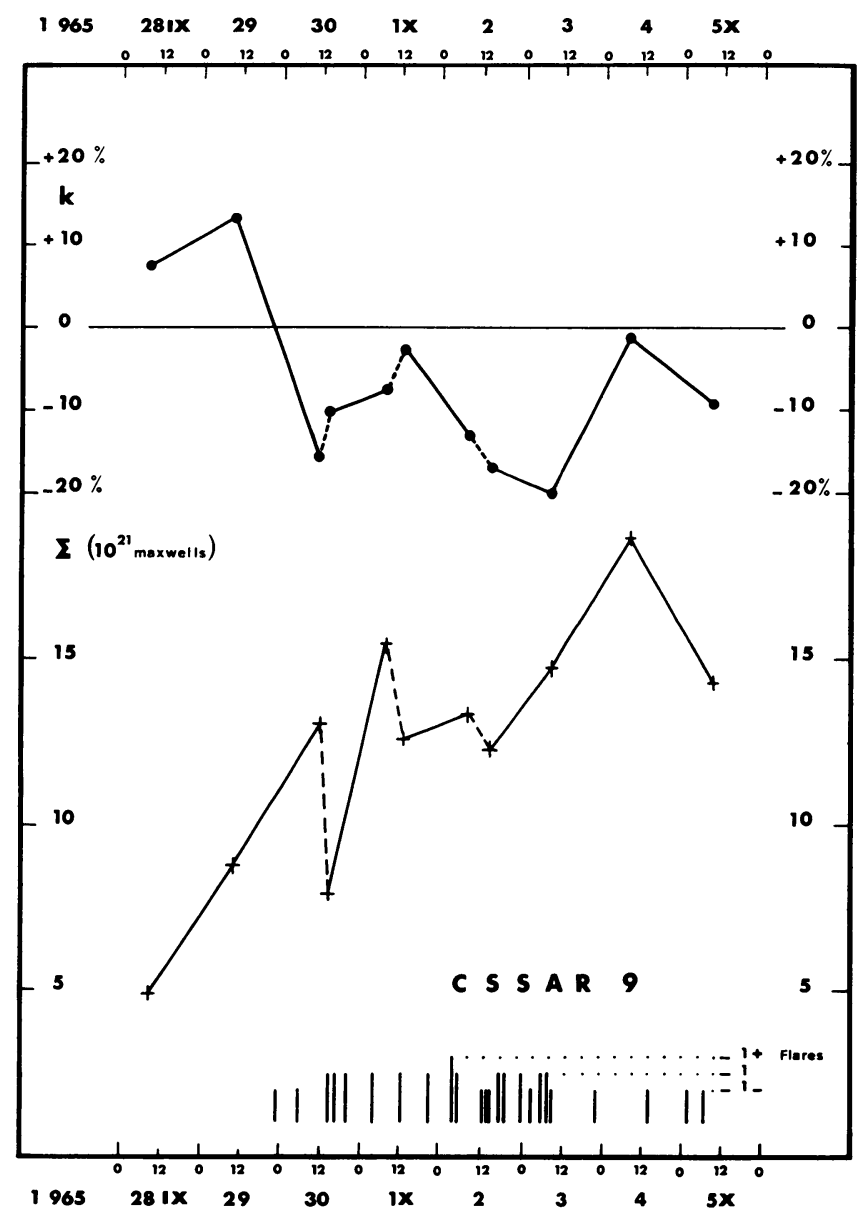

FIG. 2. The run of magnetic flux indices $\Sigma$ and $k$ (see text) for AR CSSAR 9 (September 28, October 5, 1965) as derived from 9 magnetic maps from K.A.O. and 2 from Meudon. These indices are corrected for foreshortening on the assumption that they are proportional to cos $\theta$. We observe a general increase of $\Sigma$ in agreement with the increasing size and spots number of the region, and irregular variations of $k$ : the most prominent is a change of sign between September 29 and 30, the $N$ polarity showing the larger flux before and the smaller after. This change may be related to the birth of new important spots in the region.

and dying during the history of the group. Therefore the concept of 'evolutionary features' was introduced: an 'evolutionary feature' (EF) is a sunspot or aggregate of spots inside the group, with well-defined birth and disappearance. Except for small relative motions it keeps its location in the overall structure. Figure 3 shows a summary of the history of sunspot group CSSAR 7.

The study of flare location relative to spotted EF in CSSAR 7 during its rise to maximum gave the following indications (see Figure 4). 


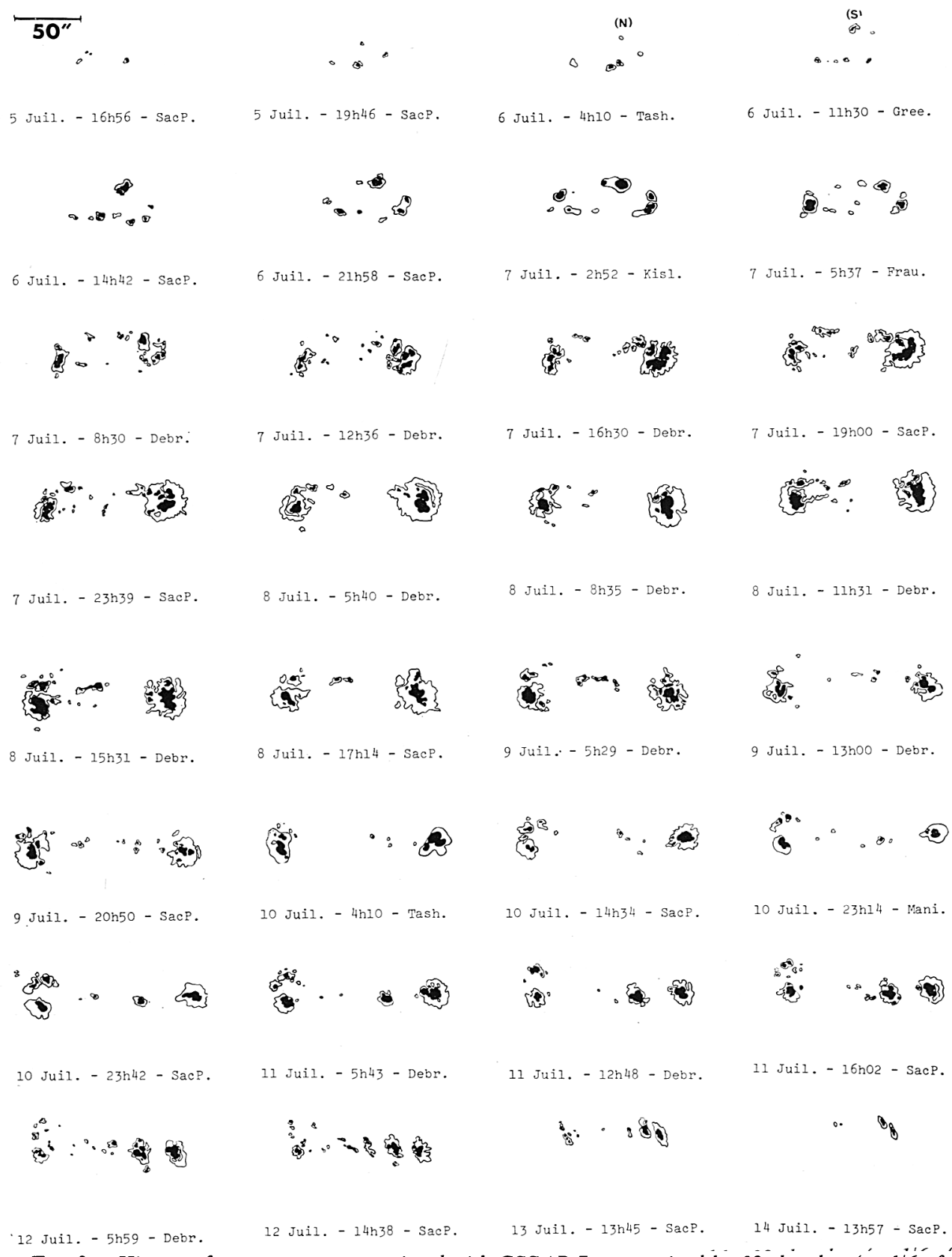

FIG. 3. History of sunspot group associated with CSSAR 7 summarized by 32 sketches (or $1 / 6$ of the total number of drawings used in the present study!). The names of observatories contributing the original photographs are abbreviated by their first four letters. East is at the left, North at top. - Note the frequent formation, evolution and disappearance of minor spots, or spots aggregates referred to in text as 'evolutionary features'. 


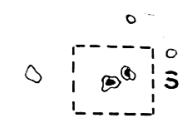

6 Juil. - 4hlo

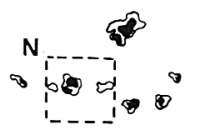

6 Juil. - 14 h42

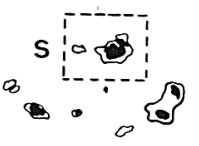

6 Juil. $-21 h 58$

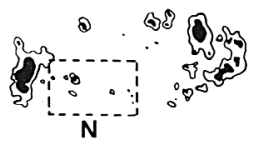

7 Juil. - 9h38

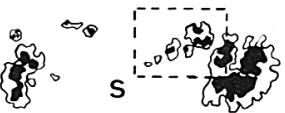

7 Juil. - 16h30

$$
\begin{aligned}
& 8
\end{aligned}
$$

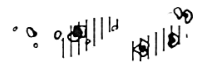

6 Juil. - 15 hl 7

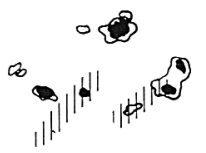

6 Juil. - 2lh58

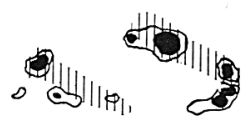

7 Juil. - 2h52

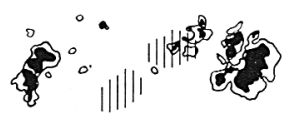

7 Juil. - $14 \mathrm{~h} 38$

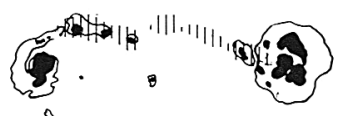

$$
8 \text { Juil. - } 3 \text { h5l }
$$

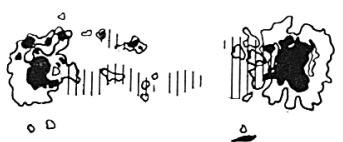

8 Juil. -7 h33

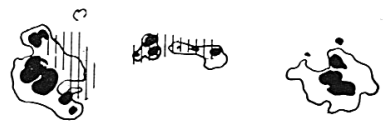

6 Juil. - 16h4l

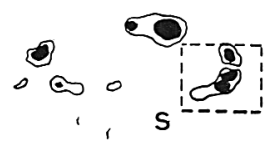

7 Juil. - 2h52

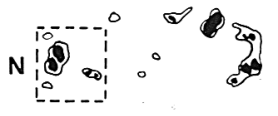

7 Juil. - 6h23

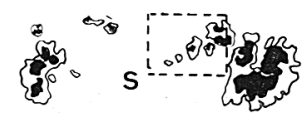

7 Juil. - 16h30

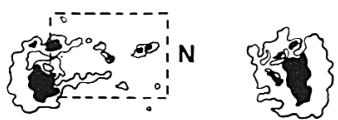

8 Juil. - Ilh31
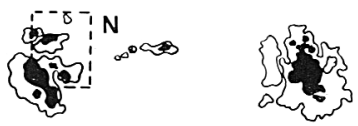

9 Juil. - 4h29

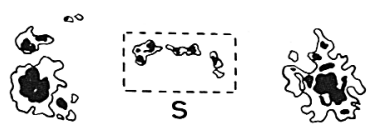

8 Juil. - 16h33

9 Juil. - 5 h29

FIG. 4. Sketches of 7 flares occurring in region CSSAR 7 (central column) in association with sunspot structure some time before (left) and after the flare (right). Each flare knot seems associated with a distinct spotted EF, each of opposite polarity and opposite sense of evolution. The involved features in each case are enclosed in a dashed square, the decreasing EF on the left pre-flare drawing, and the increasing EF on the right post-flare drawing. 
(1) Flares were made up of two brightenings, each covering a different EF.

(2) The two spotted EF involved in flares were of opposite magnetic polarities, a result in agreement with our previous finding on the distribution of flare bright knots on both sides of an inversion line.

(3) Of two spotted EF involved in a flare one was increasing in area and the other decreasing. This interesting new result may be related to our finding of variations in the index $k$ of unbalance of polarities. It emphasizes the probable relation of flares with finite changes of the magnetic structure: One might visualize the pre-flare situation as a system of coronal particles trapped in the tube of force connecting two poles: if the strengths of these two poles varies in opposite directions, the trap might eventually collapse and large precipitation phenomena, i.e. flares, will occur.

When the region CSSAR 7 had reached its maximum, flares began to occur outside spots; the brightenings were located on opposite polarities close to the inversion line of $H_{\|}$, but the available magnetic maps did not permit to evaluate variations of these polarities in order to check the above result (3).

It should be noted that the location of the bright details of the $\mathrm{H} \alpha$ plage (even outside flares) is closely related to sunspot evolution, at least in the pre-maximum phase of the AR. Clear changes of the $\mathrm{H} \alpha$ structure are preceded by the appearance of a new EF in the spotgroup. Since the brightest features in $\mathrm{H} \alpha$ have the same relationships to magnetic structure as flares proper, and also are the seat of flares, it is tempting to suggest that they are due to a permanent precipitation process of which the flare is a spectacular enhancement.

\section{References}

Giovanelli, R. (1939) Astrophys. J., 89, 555.

Gopasyuk, S., Ogir, M., Severny, A., Shaposhnikova, E. (1963) Izv. krymsk. Astrofiz. Obs., 29, 15.

Martres, M.-J., Michard, R., Soru-Iscovici, J. (1966) Ann. Astrophy's., 29, 245.

Michard, R., Mouradian, Z., Semel, M. (1961) Ann. Astrophys., 24, 54.

Severny, A. (1958) Izv. krymsk. Astrofiz. Obs., 20, 22.

Severny, A. (1960) Izv. krymsk. Astrofiz. Obs., 22, 12.

Severny, A. (1963) Izv. krymsk. Astrofiz. Obs., 30, 161.

Severny, A. (1964) Izv. krymsk. Astrofiz. Obs., 31, 159. 